\title{
Mayer, T., \& Weinacht, P.-L. (Hrsg.) (2021). Gewaltenteilung. Grundsätzliches - Historisches - Aktuelles
}

\author{
Stuttgart: Franz Steiner Verlag. 211 S., ISBN: 978-3-515-13018-9, \\ $€ 46,-$.
}

\section{Dirk Freudenberg}

Eingegangen: 28. Juni 2021 / Angenommen: 2. August 2021 / Online publiziert: 7. September 2021 (C) Der/die Autor(en) 2021

Das Verhältnis der staatlichen Gewalten Legislative, Exekutive und Judikative entspringt den Beziehungen von Recht und Politik und dem uralten Kampf um das Recht. Aktuell spiegelt sich dieser Kampf in der Fragestellung, ob die Pandemie ,die Stunde der Exekutive“ oder ,die Stunde der Legislative“ ist. Damit ist das Verhältnis von Regierung und Parlament angesprochen in der Frage, wo die Verantwortlichkeiten für Entscheidungen in der Stunde der Not liegen, und in wieweit die Regierungen in Bund und in den Ländern ermächtigt sind, Maßnahmen zum Schutz der Bevölkerung zu ergreifen, ohne dass damit verbundene (Grundrechts-)Einschränkungen vom Bundestag und ggf. vom Bundesrat mitgetragen werden müssen, und nicht der (nachträglichen) Korrektur der Gerichte bedürfen. Es handelt sich also im Kern um einen Machtkampf zwischen Regierung und Parlament und ist dem Grunde nach ein klassisches staatswissenschaftliches Problem im Verhältnis der Staatsgewalten zueinander - wenn man unter Staatswissenschaft Politikwissenschaft, Rechtswissenschaft sowie Soziologie und Philosophie im Sinne eines integrativen Selbstverständnisses mit einem interdisziplinären Ansatz versteht. Genau diesem Ansatz folgen die Herausgeber, indem sie Autoritäten der unterschiedlichen Disziplinen in dem vorgestellten Band versammelt haben, welche aus den jeweils unterschiedlichen Blickwinkeln ihrer wissenschaftlichen Disziplin Aspekte zum Thema aufgreifen und so insgesamt an ausgewählten Akteuren und Beispielen einen ideengeschichtlichen Überblick von der Antike bis hin zu aktuellen Staatsverfassungen sowie Spielarten in den Verfassungswirklichkeiten ausgewählter Staaten geben.

Den Auftakt macht Klaus Rosen, der die Themenstellung anhand des antiken Stadtstaats Athen und des späteren römischen Weltreiches behandelt. Paul-Ludwig Weinacht stellt mit Montesquieu den Klassiker politischen Denkens in den Mit-

Dr. Dirk Freudenberg $(\bowtie)$

Bundesakademie für Bevölkerungsschutz und Zivile Verteidigung (BABZ), Bundesamt für

Bevölkerungsschutz und Katastrophenhilfe (BBK), Bonn, Deutschland

E-Mail: Dirk.Freudenberg@bbk.bund.de 
telpunkt seiner Darstellung und untersucht dessen Rezeption in Deutschland im 19. und 20. Jahrhundert, zuletzt in der Neuverfassung westdeutscher Länder nach dem Zweiten Weltkrieg. Hans-Christof Kraus blickt in seinem Beitrag auf die Gewaltenbeschränkung und das Prinzip der „Checks und Balances“ sowie der Parlamentssouveränität in Großbritannien. Reinhard Zintl untersucht das Prinzip der Gewaltenteilung an den USA und damit an einem Klassiker der neuzeitlichen Demokratien. Barbara Zehnpfennig kontrastiert das Prinzip der Gewaltenteilung gegenüber der Konzentration der Gewalten im totalitären System am Beispiel der beiden totalitären Regime im Deutschland des 20. Jahrhunderts. Margareta Mommsen stellt demgegenüber am Beispiel Russlands unter Präsident Wladimir Putin die Gewaltenteilung in der ,gelenkten Demokratie“ dar. Nikolaus Werz untersucht das Thema am Beispiel lateinamerikanischer Staaten. Gérard-François Dumont stellt in seinem französischsprachigen Beitrag dar, wie sich die Staatsmacht Frankreichs unter Präsident Emmanuel Macron insbesondere in ihrer programmatischen Ausrichtung gegenüber Europa gestaltet. Hans-Joachim Veen widmet sich dem Problem der Amtszeitbegrenzungen als einer Komponente des Gewaltenteilungsprinzips und dem Phänomen ,„ewiger Kanzlerschaften“ am Beispiel der Bundesrepublik Deutschland. Im Mittelpunkt der Untersuchung Tilmann Mayers steht Max Webers Konzept der Führerdemokratie. Detlev Fischer analysiert die Stellung der Judikative in der Bundesrepublik Deutschland vor allem mit Blick auf die Verfassungsgerichtsbarkeit. Dietrich Murswiek betrachtet in seinem Beitrag vornehmlich die Verteilung der Gewalten auf unterschiedliche Ebenen der Europäischen Union und fokussiert hierbei insbesondere auf die Zuständigkeitsverteilung zwischen der Union und den einzelnen Mitgliedsstaaten, wobei er die grundlegende Bedeutung des Prinzips der Subsidiarität für die Kompetenzverteilung besonders herausstellt. Hans-Joachim Lauth gibt am Ende des Kompendiums eine Zusammenfassung der Entwicklung der Gewaltenteilung im 20. Jahrhundert aus der Perspektive der vergleichenden Politikwissenschaft.

Das vorliegende Werk ist im staatswissenschaftlichen Kontext für Wissenschaft und Lehre, aber unter Umständen auch für die Beschäftigten in der politischen Administration und Verwaltung mit einem tiefergehenden Interesse an ideengeschichtlichen Hintergründen und Zusammenhängen von großem Wert, weil Erkenntnisse aufgeworfen werden, die helfen können, aktuelle Zusammenhänge sowie die Beziehungen von Politik und Recht sowie den Kampf ums Recht besser zu verstehen.

Funding Open Access funding enabled and organized by Projekt DEAL.

Open Access Dieser Artikel wird unter der Creative Commons Namensnennung 4.0 International Lizenz veröffentlicht, welche die Nutzung, Vervielfältigung, Bearbeitung, Verbreitung und Wiedergabe in jeglichem Medium und Format erlaubt, sofern Sie den/die ursprünglichen Autor(en) und die Quelle ordnungsgemäß nennen, einen Link zur Creative Commons Lizenz beifügen und angeben, ob Änderungen vorgenommen wurden.

Die in diesem Artikel enthaltenen Bilder und sonstiges Drittmaterial unterliegen ebenfalls der genannten Creative Commons Lizenz, sofern sich aus der Abbildungslegende nichts anderes ergibt. Sofern das betreffende Material nicht unter der genannten Creative Commons Lizenz steht und die betreffende Handlung nicht nach gesetzlichen Vorschriften erlaubt ist, ist für die oben aufgeführten Weiterverwendungen des Materials die Einwilligung des jeweiligen Rechteinhabers einzuholen.

Weitere Details zur Lizenz entnehmen Sie bitte der Lizenzinformation auf http://creativecommons.org/ licenses/by/4.0/deed.de. 